\title{
Surfactant concentration and its effect on the flow field inside an oil droplet rising in an aqueous medium: LIF and PIV measurements
}

\author{
A. C. Bishop, P. J. Thomas \& P. Denissenko \\ School of Engineering, University of Warwick, UK
}

\begin{abstract}
We explore the effects of adsorption of a fluorescent surfactant-like substance (Rhodamine-6G) to the interface of a droplet of mineral oil rising through a waterglycerol mixture with $\operatorname{Re} \ll 1$. As Rhodamine- $6 \mathrm{G}$ adsorbs to the interface it collects in a stagnant cap at the base of the droplet. This in turn impedes the internal flow of the droplet. Laser-Induced Fluorescence (LIF) and Particle Image Velocimetry (PIV) are used as measurement techniques to explore the effects of Rhodamine-6G adsorbing to the oil-aqueous interface.

LIF is used to observe the transport of surfactant (Rhodamine-6G) as it adsorbs to the front facing section of the droplet interface and desorbs in the wake behind the droplet. The LIF visualisation shows the accumulation of Rhodamine-6G at the rear of the droplet in the form of a cap, as well as the formation of a surfactantdepleted tail behind the droplet. This cap grows in size as the droplet rises and develops at different rates for different concentrations of Rhodamine-6G. PIV measurements are obtained to visualise the circulation inside the droplet and to measure the angle of the stagnant cap as the droplet rises, with these measurements being linked to the LIF results.

Keywords: interface, PIV, LIF, surfactant, adsorption, Rhodamine-6G, stagnant cap, oil droplet.
\end{abstract}

\section{Introduction}

This paper will look at experimental methods for examining the adsorption of a surface active agent (surfactant) to a buoyant droplet of oil. Firstly, LaserInduced Fluorescence (LIF) will be used to examine the interface and outside of 


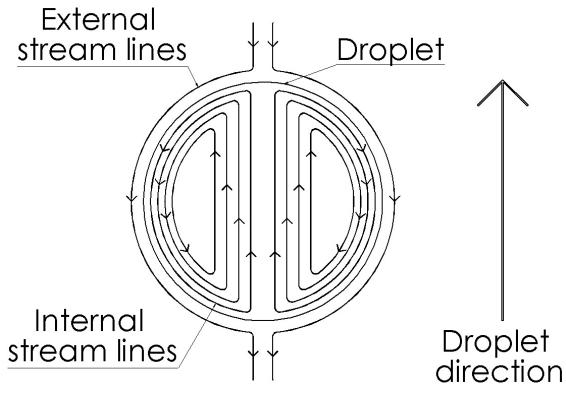

Figure 1: Laminar flow past a droplet. The fluid of the external flow pushes the interface in the direction of the flow until the internal droplet fluid meets at the base and recirculates up through the centre, forming eddies.

the droplet. This is made possible by the use of a fluorescent surfactant which doubles as a tracer. Following this, the oil droplet will be seeded with reflective particles to enable Particle Image Velocimetry (PIV) to be performed. PIV allows the visualisation of the internal flow of the droplet. This enables the examination of the effect that surfactant adsorbing to the interface has on this internal flow structure. These two techniques work together to give a full understanding of the effects of adsorption of a surfactant on a rising droplet of oil.

This paper will initially present background information on the circulation of a droplet in a flow field, as well as the effect that the adsorption of surfactant will have on the mobility of the interface of a droplet. This will be followed by the presentation of the experimental setup for both techniques, as well as methods used to analyse and understand the data obtained from these experiments. The results will be presented with an effort to tie the two techniques together, before moving on to a discussion and conclusions.

\section{Background}

A droplet in a fluid subjected to an external flow will have internal circulation (McDonald [1]). This is caused by the fluid on the interface of the droplet being accelerated to match the speed of the flow (Fig. 1). This figure demonstrates laminar flow, which is the regime in which these experiments will occur. As the internal fluid is pushed down it gets recirculated up the centre of the droplet forming eddies.

The introduction of an admixture (in this case surfactant) to the interface can disrupt these eddies. Any surfactant on the surface will be swept around the interface of the droplet to the rear, where, if it has no way to break free of the interface, it will accumulate and form a stagnant cap (Fig. 2) (Chen and Lee [2]). This hinders the flow within the droplet and the internal eddies shrink. A droplet completely covered in surfactant would have a physical barrier around the interface - a Gibbs monolayer (Hiemenz and Rajagopalan [3]), so would behave as a rigid 


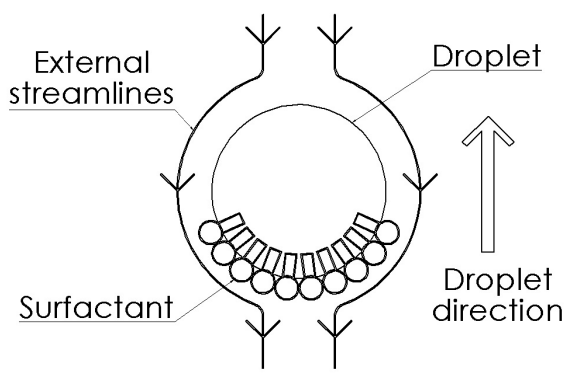

Figure 2: Formation of rigid surfactant stagnant cap in flow. As more of the surfactant gathers at the rear of the droplet, it must extend up the sides and forms a physical barrier between the fluid in the flow and that in the droplet.

body. However when in a flow field, the surfactant is swept away from the front of the droplet to the rear, breaking the physical barrier. The fluid in the front of the droplet matches the fluid in the external flow while there is still a barrier impeding flow at the rear.

The tracer used in this case is a surfactant-like substance, Rhodamine-6G. A surfactant has the unique property of being both hydrophobic and hydrophillic (Atkins and de Paula [4]), making its equilibrium positioning at an interface. Rhodamine-6G has the properties of a very weak surfactant (Paul et al. [5]); it will disperse in an aqueous phase and will move to the interface between the aqueous phase and an oil phase, but will not disperse into the oil phase as the hydrophilic segment is stronger.

\section{Experimental setup}

This experiment consisted of passing a droplet of oil through a tank filled with an aqueous medium. Mineral oil was used, injected with a syringe at the base of the tank to form a droplet approximately $4 \mathrm{~mm}$ in diameter. The aqueous medium was index-matched to the oil, and consisted of a mixture of $96 \%$ glycerol and $4 \%$ water. A florescent surfactant, Rhodamine- $6 \mathrm{G}$, was dispersed throughout the aqueous medium and adsorbs to the interface of the oil droplet as it rises through the solution. A vertical light sheet was passed through the centre of the droplet at ten different heights enabling visualisation of the Rhodamine- $6 \mathrm{G}$ adsorbing to the interface over time. This setup is demonstrated in Fig. 3.

The high viscosity of the glycerol keeps the rising velocity low. This gives a low Reynolds number ( $\operatorname{Re} \sim 0.037)$ and so ensures laminar flow. Images are obtained every $57 \mathrm{~mm}$ with four different concentrations of Rhodamine-6G. The concentrations used are $2.5 \times 10^{-4} \mathrm{~g} / 1,1.25 \times 10^{-4} \mathrm{~g} / 1,0.625 \times 10^{-4} \mathrm{~g} / \mathrm{l}$, and $0.3125 \times 10^{-4} \mathrm{~g} / \mathrm{l}$. 


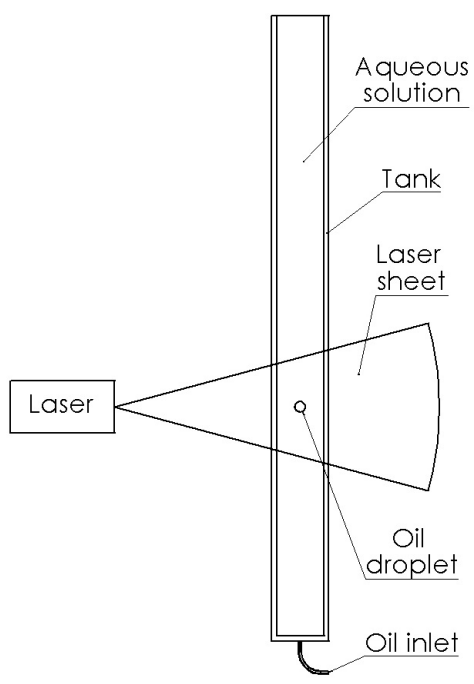

Figure 3: Experimental setup.

\subsection{LIF setup and analysis}

LIF data was obtained due to the fluorescent properties of Rhodamine-6G molecules. Rhodamine-6G fluorescence is activated by the adsorption of green laser light (wavelength $532 \mathrm{~nm}$ ), and releases light detected using a $550 \mathrm{~nm}$ wavelength long pass filter (Arden et al. [6]). Raw data was gathered in the form of photographs captured by a camera set up perpendicularly to the light sheet.

A normalisation process was used to remove any irregularities from the image, after which the data was processed to numerically show the volume of Rhodamine-6G around the interface of the droplet. The analysis of this data was performed in Matlab R2012b by measuring the light intensity of the pixels in the captured image. The droplet is easily identifiable in the image as no Rhodamine-6G enters the oil phase and so it remains dark. The intensities of the pixels around the interface were measured and linked to the number of molecules, with brighter patches indicating a build-up of Rhodamine-6G. This enables the distribution of Rhodamine- $6 \mathrm{G}$ around the interface to be measured as well as the total Rhodamine-6G on the droplet.

\subsection{PIV setup and analysis}

To gather PIV data of the internal flow of the droplet the oil was seeded with silver coated reflective hollow glass spheres, $10 \mu \mathrm{m}$ in diameter. A $532 \mathrm{~nm}$ wavelength long pass filter was used to capture the light from the laser reflected by the particles. The concentration of particles was kept at a minimum to reduce any interference effects that the particles may have on the interface. A sequence of 


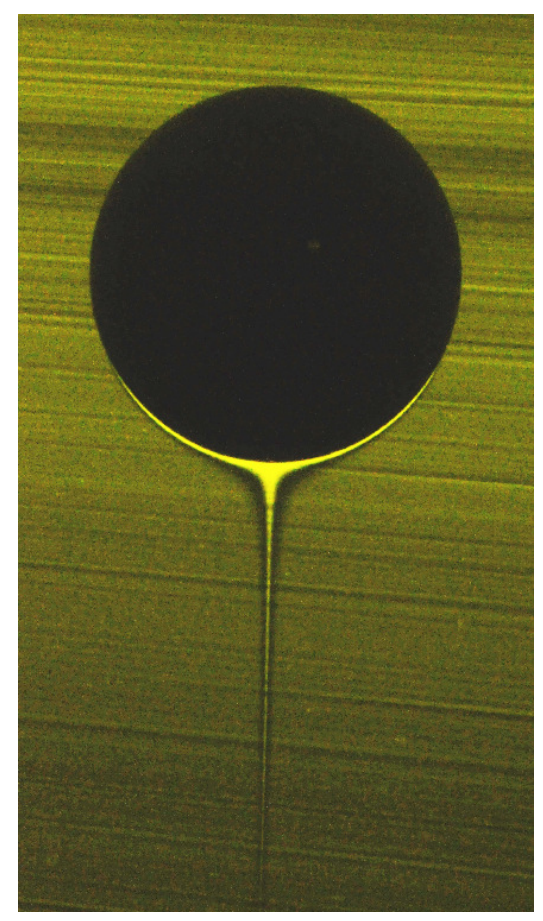

Figure 4: Raw data obtained from LIF. This droplet is $456 \mathrm{~mm}$ from the base of the tank with an initial bulk Rhodamine-6G concentration of $0.625 \times 10^{-4} \mathrm{~g} / \mathrm{l}$. The Rhodamine- $6 \mathrm{G}$ gathers in a bright cap around the base of the dark droplet.

images are required for PIV analysis so a camera capable of recording at $60 \mathrm{~Hz}$ was used.

Initial PIV analysis was performed in Dantec Dynamics' DynamicStudio v4.10 software. An adaptive PIV technique is used to track the particles and give a flow pattern within the droplet (Dantec Dynamics [7]). This allows the internal eddies of the droplet to be visualised. This data is exported to Matlab R2012b in which the velocity around the interface is examined and the development of the stagnant cap is mapped.

\section{Results}

\subsection{LIF results}

An example of the raw data is given in Fig. 4. The key points to observe are that the droplet remains dark, confirming no Rhodamine-6G crosses the boundary 


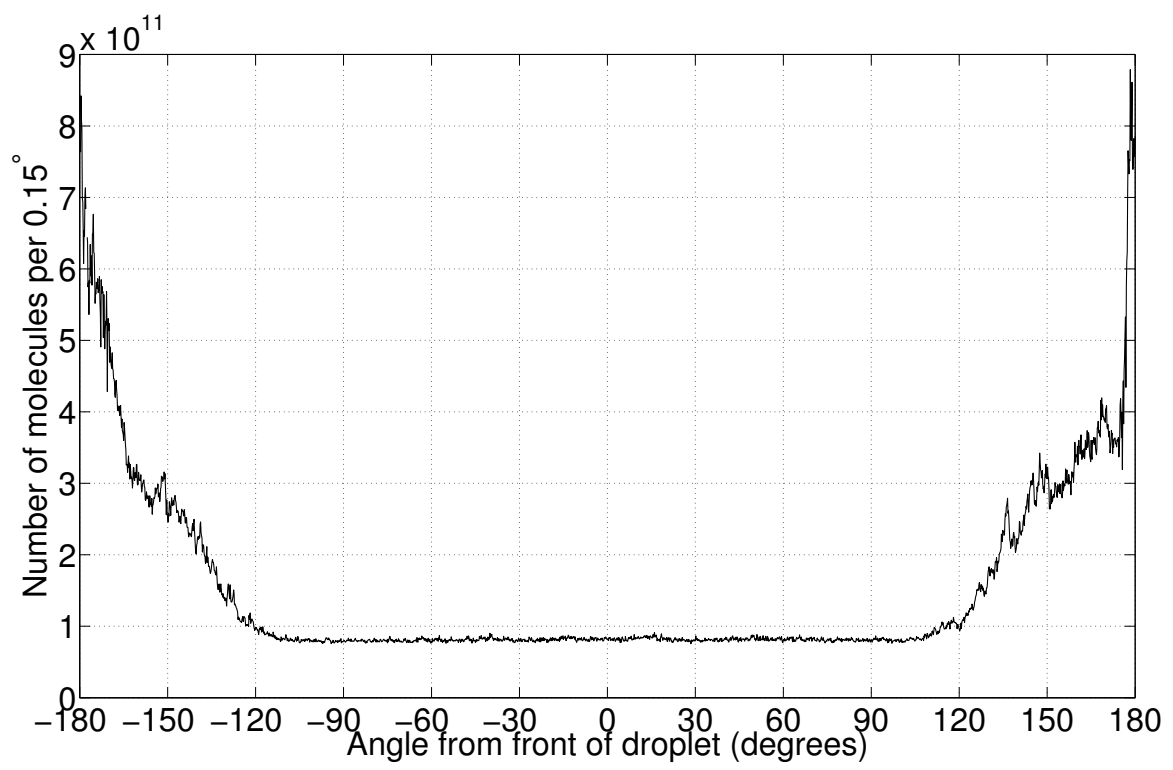

Figure 5: Total amount of Rhodamine-6G around a droplet with Rhodamine-6G concentration of $0.625 \times 10^{-4} \mathrm{~g} / \mathrm{l}$ and height from base of tank of $456 \mathrm{~mm}$. The angle is measured from the front point of the droplet. The number of molecules is measured radially outwards from the droplet until equal to the bulk concentration for each $0.15^{\circ}$ segment.

interface. Visually no Rhodamine-6G can be seen to be gathering on the forwards face of the droplet. However, adsorption is occurring and this can be confirmed by the existence of a dark wake behind the droplet. As this is darker than the surrounding bulk solution it indicates that this area is depleted of Rhodamine-6G, which has instead been adsorbed to the interface. There is a bright cap around the base of the droplet. This indicates a build-up of Rhodamine-6G, due to the external flow transporting surfactant down the sides of the droplet to the rear. There is a bright tail within the depleted wake. This is due to the surfactant saturation level being reached in the rear of the droplet, and so Rhodamine- $6 \mathrm{G}$ is washed from the rear of the droplet back into the external flow to form a surfactant-rich wake.

Fig. 5 shows a profile of the distribution of Rhodamine-6G around the droplet. There is a very low level of Rhodamine- $6 \mathrm{G}$ around the front $240^{\circ}$ of the droplet $\left(-120^{\circ}\right.$ to $\left.120^{\circ}\right)$. Around the rear $120^{\circ}$ of the droplet the number of molecules grow at a steady rate before rapidly increasing to a peak $\left(120^{\circ}\right.$ to $\left.-120^{\circ}\right)$. The steady growth corresponds to the bright cap in Fig. 4, with the peak at 180 degrees corresponding to the surfactant-rich tail.

By integrating the curve in Fig. 5, the total number of Rhodamine-6G molecules on the droplet can be calculated. This enables a graph of the number of 


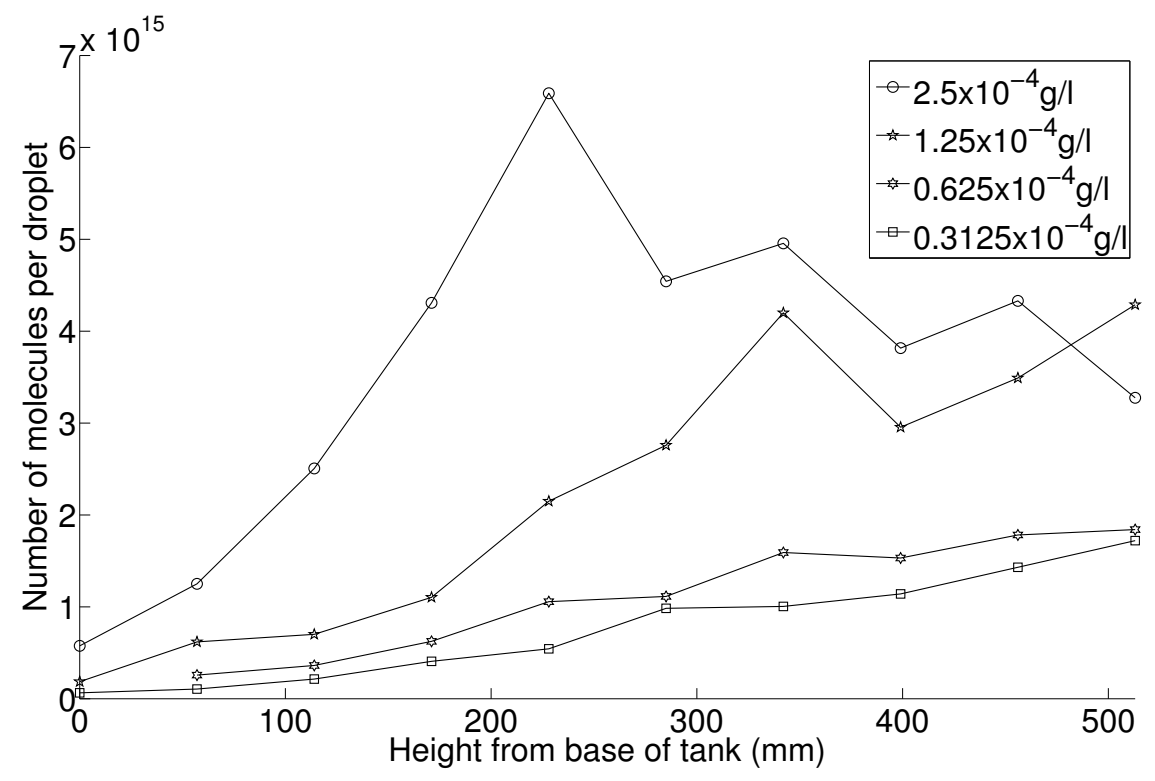

Figure 6: Number of Rhodamine-6G molecules at the interface around the rear $120^{\circ}$ of the droplet for different Rhodamine- $6 \mathrm{G}$ bulk concentrations.

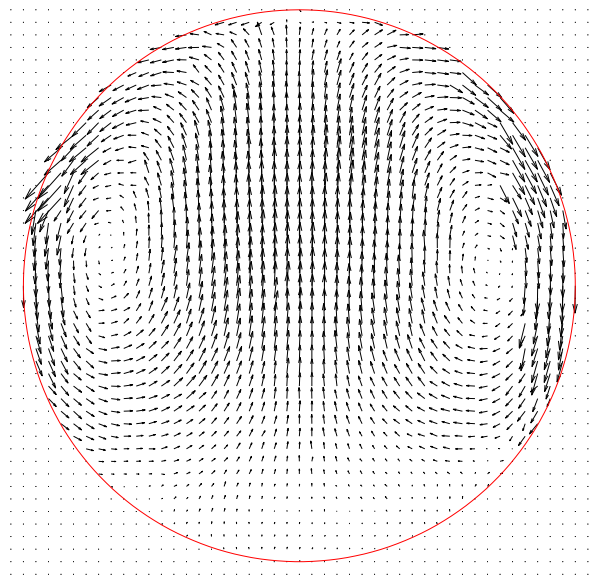

Figure 7: PIV velocity field of the internal flow of a droplet at a height of $456 \mathrm{~mm}$ from the base of the tank with Rhodamine-6G concentration of $0.625 \times 10^{-4}$. 


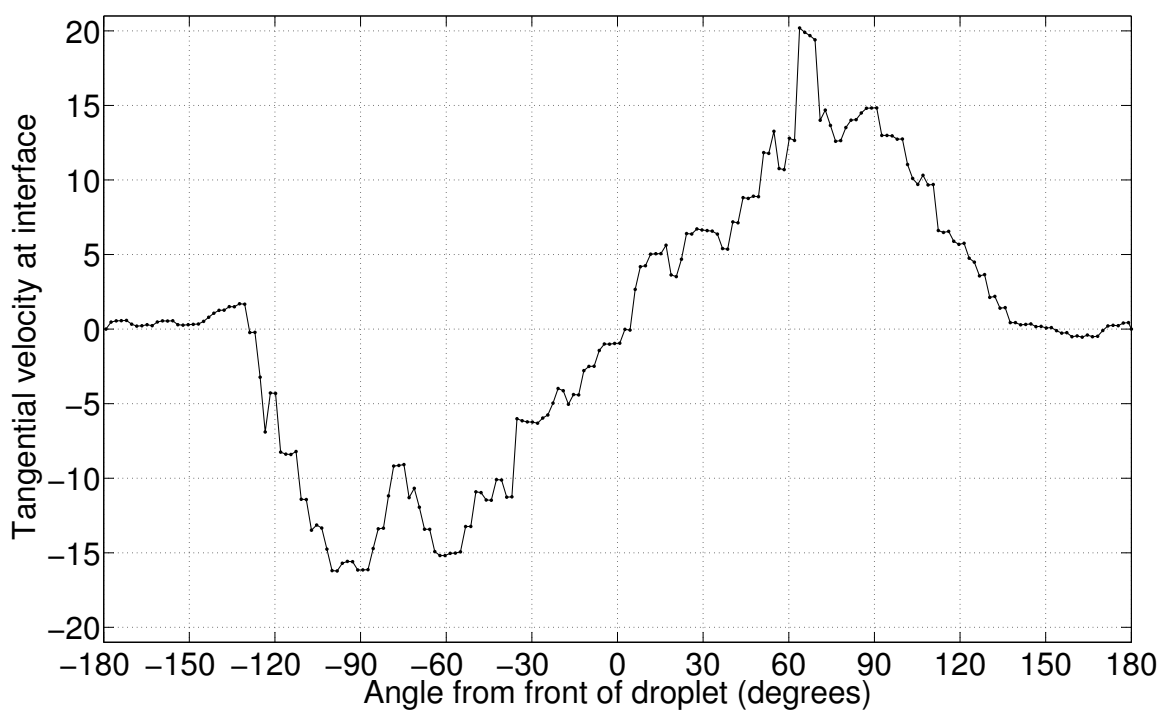

Figure 8: Tangential velocity at the droplet interface at a height of $456 \mathrm{~mm}$ from the base of the tank with Rhodamine- $6 \mathrm{G}$ concentration of $0.625 \times 10^{-4}$.

Rhodamine-6G molecules against the height of the droplet in the tank to be plotted and Rhodamine-6G concentrations can be compared (Fig. 6). For Rhodamine-6G concentration of $2.5 \times 10^{-4} \mathrm{~g} / \mathrm{l}$ the number of molecules in the tail rises steadily to a peak at $228 \mathrm{~mm}$ from the base of the tank before levelling off. This shows the point at which the droplet reaches the surfactant saturation level. At this point the surfactant is being washed back into the bulk at the same rate of adsorption to the interface. At the lower Rhodamine- $6 \mathrm{G}$ concentration of $0.3125 \times 10^{-4} \mathrm{~g} / \mathrm{l}$ there is a maintained steady increase of Rhodamine- $6 \mathrm{G}$ over the height of the tank; the surfactant saturation level is not reached. This demonstrates that the concentration of the surfactant in the bulk solution is the driving force behind the development of the stagnant cap at comparative heights.

\subsection{PIV results}

A sequence of images captured at $60 \mathrm{~Hz}$ was run through an adaptive PIV algorithm in DynamicStudio to give the velocity profile of the internal flow of the droplet (Fig. 7).

This profile shows two eddies circulating at the top of the droplet, symmetrically around the vertical axis. This is to be expected due to the direction of the external flow. The droplet boundary is pushed downwards, reaching its peak speed in the top half of the droplet (shown by the magnitude of the vectors). The flow continues 


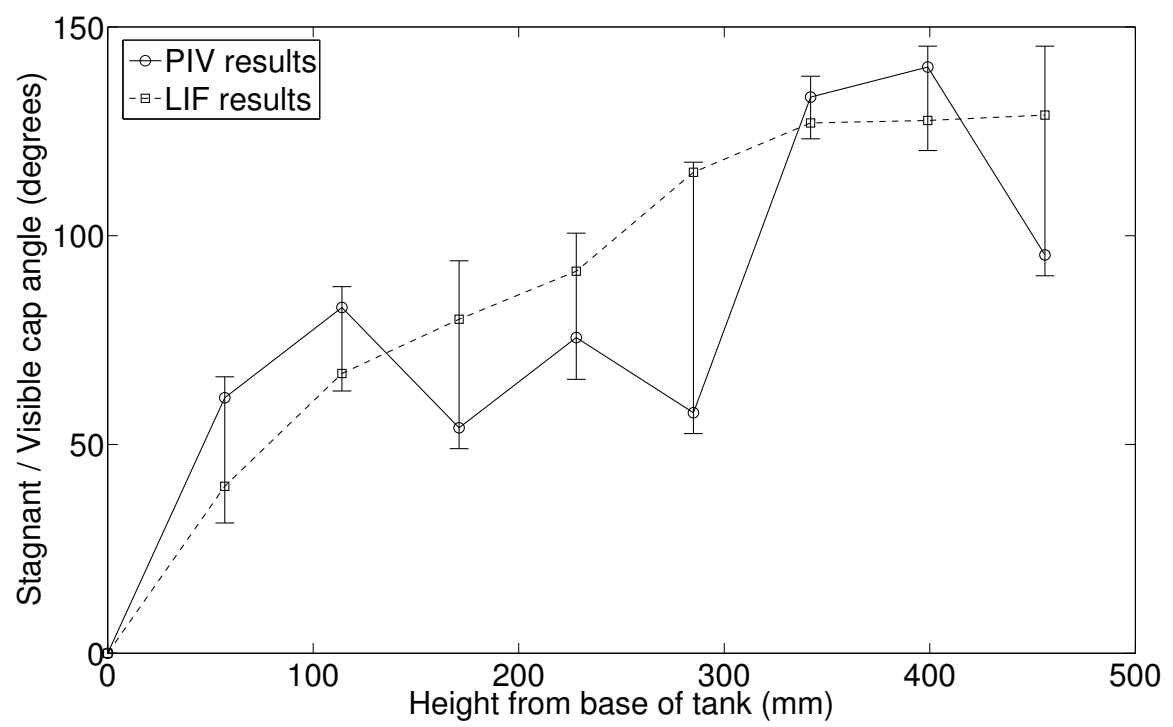

Figure 9: Graph of cap size in relation to height of droplet with error bars for PIV uncertainties. Rhodamine- $6 \mathrm{G}$ concentration of $0.625 \times 10^{-4}$.

back up the centre line of the droplet. The vectors in the bottom half of the droplet have a very small magnitude. This signifies that the fluid is this region of the droplet forms a stagnant region. When visually comparing this to Fig. 5 this stagnant region corresponds to the external area with a build-up of Rhodamine6G. The build-up of Rhodamine-6G on the interface creates a physical barrier, impeding the internal flow, leading to this stagnant region within the droplet.

To find where on the interface of the droplet this stagnant region begins, the profile of Fig. 7 is processed to give a graph of the velocity at the interface against the angle from the front of the droplet (Fig. 8).

There is a stagnant point at the front of the droplet $\left(0^{\circ}\right)$, from which the velocity increases to its maximums at $+63.8^{\circ}$ and $-89.2^{\circ}$. The velocity drops down to practically zero at $+137.6^{\circ}$ and $-128.8^{\circ}$. This leaves a region of $93.6^{\circ}$ at the rear of the droplet where the velocity is close to zero corresponding to the stagnant cap region.

In this way the arc of the stagnant cap was calculated for each height for this Rhodamine-6G concentration (solid line, Fig. 9). The stagnant cap grows with height from the base of the tank as expected. The dashed line on the graph corresponds to the LIF data. The LIF data was obtained by measuring the arc where Rhodamine- $6 \mathrm{G}$ concentration was greater than that of the bulk solution for each height. Fig. 9 shows that the general trend for the PIV data matches that of the LIF data. 


\section{Conclusions}

This paper has shown the effect of Rhodamine- $6 \mathrm{G}$ adsorption on the behaviour of a rising oil droplet. Two different methods have been explored in order to measure both the effects of a surfactant-like admixture on the formation of a stagnant cap at the rear of the droplet and the internal flow of the droplet. The LIF data give a clear picture of the transportation of Rhodamine-6G to the rear of the droplet where it gathers forming a stagnant cap. They show that the initial concentration of Rhodamine-6G in the bulk solution is the driving force behind the speed of development of the stagnant cap. The PIV data gathered supports the findings of the LIF data by examining the internal flow of the droplet. Visualisation of the flow inside the droplet revealed eddies as well a stagnant area. This stagnant area grows in size as the droplet rises showing that a growing stagnant cap is impeding the internal flow. The cap angle has been tracked over height and compared to the data gathered by LIF. These methods support each other in detecting the effects that a growing amount of Rhodamine-6G gathering at the rear of a droplet have on the interface and internal flow of a droplet.

\section{Acknowledgement}

This study was supported by the EPSRC.

\section{References}

[1] McDonald, J., The shape and aerodynamics of large raindrops. Journal of Meteorology, 11, pp. 478-494, 1954.

[2] Chen, L.H. \& Lee, Y.L., Adsorption behaviour of surfactants and mass transfer in single-drop extraction. Americal Institute of Chemical Engineers, 46(1), pp. 160-168, 2000.

[3] Hiemenz, P. \& Rajagopalan, R., Principles of Colloid and Surface Chemistry. Marcel Dekker: New York, 3rd edition, 1997.

[4] Atkins, P. \& de Paula, J., Elements of Physical Chemistry. Oxford University Press: Oxford, 5th edition, 2009.

[5] Paul, P., Hussain, S., Bhattacharjee, D. \& Pal, M., Adsorption of cationic laser dye onto polymer/surfactant complex film. Chinese Journal of Chemical Physics, 24(3), pp. 348-352, 2011.

[6] Arden, J., Deltau, G., Huth, V., Kringel, U., Peros, D. \& Drexhage, K., Fluorescence and lasing properties of rhodamine dyes. Journal of Luminescence, 48 to 49, Part 1, pp. 352-358, 1991.

[7] Dantec Dynamics, DynamicStudio User's Guide, 2013. 